\title{
Исследование параметров наноразмерного слоя в наногетероструктурах на основе полупроводниковых соединений $A^{\text {II }}$ BI $^{\mathrm{VI}}$
}

\author{
(C) М.Б. Караваев, Д.А. Кириленко, Е.В. Иванова, Т.Б. Попова, А.А. Ситникова, \\ И.В. Седова, М.В. Заморянская
}

Физико-технический институт им. А.Ф. Иоффе Российской академии наук, 194021 Санкт-Петербург, Россия

E-mail: estonianchameleon@gmail.com

(Получена 28 мая 2016 г. Принята к печати 24 июня 2016 г.)

\begin{abstract}
Методами локальной катодолюминесценции и рентгеноспектрального микроанализа проведено комплексное исследование широкозонных наногетероструктур на основе $\mathrm{ZnSe}$, полученных методом молекулярнопучковой эпитаксии. Было показано, что используемые методы позволяют неразрушающим способом определять глубину залегания, элементный состав и геометрические параметры наноразмерного слоя ZnCdSe. Точность результатов контролировалась методом просвечивающей электронной микроскопии. Методики исследования основаны на возможности варьирования энергии первичного электронного пучка, что приводит к изменению областей генерации характеристического рентгеновского излучения и катодолюминесценции.
\end{abstract}

DOI: 10.21883/FTP.2017.01.43996.8249

\section{1. Введение}

Актуальность создания миниатюрных лазеров, излучающих в зеленой $(\lambda=520-550$ нм $)$ области спектра, возрастает в связи с увеличивающейся потребностью в них для массового применения в проекционном лазерном и $3 D$ телевидении, всепогодных системах обеспечения навигационной безопасности транспортных средств, локальных волоконно-оптических линиях связи, медицинской флуоресцентной диагностике клеток и т.д. В связи с этим лазерные гетероструктуры на основе широкозонных полупроводников $\mathrm{A}^{\mathrm{II}} \mathrm{B}^{\mathrm{VI}}$, обладающие высокой квантовой эффективностью люминесценции в „истинном“ зеленом спектральном диапазоне, по-прежнему представляют особый интерес для создания приборов, излучающих в этой области спектра.

Продолжаются работы по совершенствованию конструкции и технологии получения вышеуказанных лазерных гетероструктур. В связи с этим разработка новых методов определения геометрических параметров выращенных структур неразрушающим способом остается по-прежнему актуальной. В данной работе показана возможность использования метода рентгеноспектрального микроанализа (РСМА) для получения информации о составе и глубине залегания наноразмерного слоя, входящего в состав гетероструктуры, и метода локальной катодолюминесценции (КЛ) для определения относительной эффективности люминесценции гетероструктуры и геометрических параметров наноразмерного слоя.

\section{2. Методика эксперимента}

Объектами исследования в данной работе являлись две гетероструктуры (\#267 и \#268), полученные методом молекулярно-пучковой эпитаксии (МПЭ) псевдоморфно на подложках GaAs (001) с использованием буферного слоя GaAs в двухкамерной установке МПЭ следующей конструкции (рис. 1):

- верхний слой $\mathrm{ZnMgSSe}$,

- сверхрешетка (CP) $(\mathrm{ZnSe} / \mathrm{ZnSSe})_{10}$,

- наноразмерный слой (HC) $\mathrm{ZnCdSe}$,

- CP $(\mathrm{ZnSe} / \mathrm{ZnSSe})_{10}$,

- слой ZnMgSSe,

- CP $(\mathrm{ZnSe} / \mathrm{ZnSSe})_{176}$,

- подложка GaAs.

Рентгеновские и катодолюминесцентные исследования проводились на электронно-зондовом микроанализаторе „Камебакс“, снабженном четырьмя рентгеновскими волновыми спектрометрами и станцией для катодолюминесцентных исследований [1]. Исследования методом просвечивающей электронной микроскопии (ПЭМ) проводились на электронных микроскопах ЕM-420 (энергия электронного пучка 100 кэВ) и JEOL $2100 \mathrm{~F}$ (энергия электронного пучка 100 кэВ).

Вариация энергии первичного электронного пучка при КЛ исследованиях и определении состава методом РСМА позволяет менять область генерации КЛ и характеристического рентгеновского излучения. В первую очередь область генерации обоих процессов определяется областью торможения электронов первичного электронного пучка. Размер ее может быть с хорошей точностью рассчитан методом Монте-Карло с использованием программы CASINO [2]. На рис. 1 представлены расчеты глубины проникновения электронов для исследованных структур.

Область генерации характеристического рентгеновского излучения всегда меньше, чем область торможения электронов первичного электронного пучка, и ограничивается областью, в которой энергия первичных электронов больше энергии возбуждения соответствующего рентгеновского излучения. В большинстве случаев для РСМА эта энергия лежит в пределах от 1 до 15 кэВ 


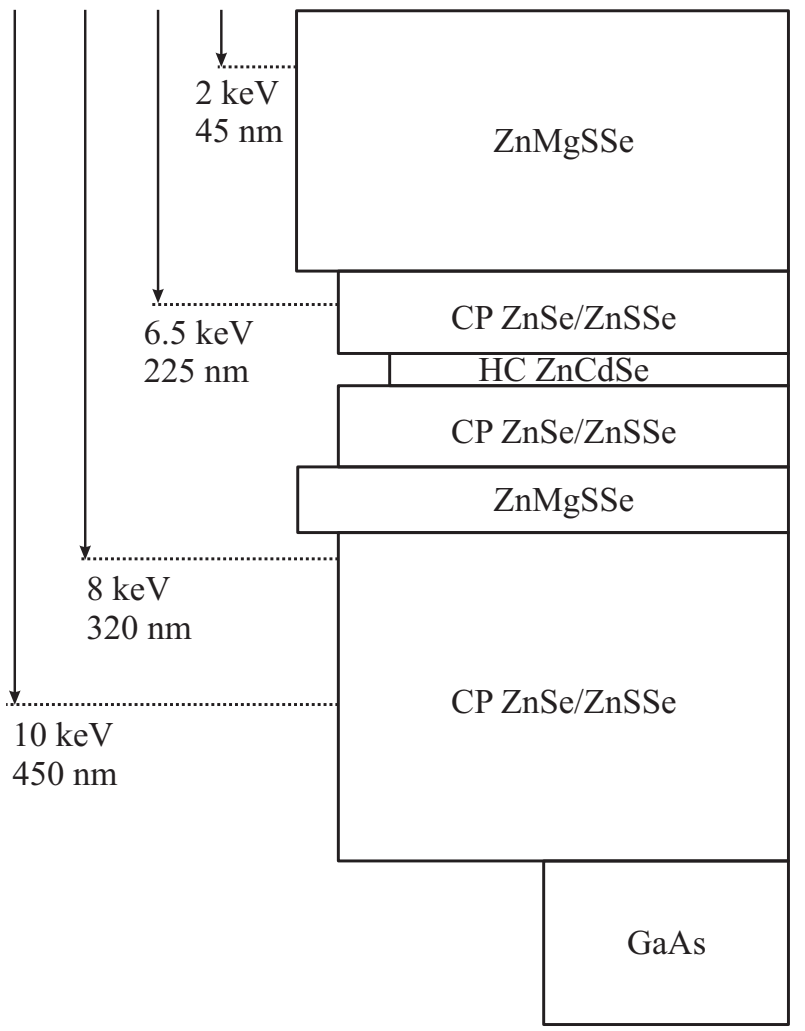

Pис. 1. Дизайн гетероструктур. Стрелками указана глубина проникновения электронов при соответствующей энергии первичных электронов.

и также может быть рассчитана с хорошей точностью в программе CASINO [2]. Метод вариации энергии первичного электронного пучка позволяет определять элементный состав слоев на глубинах от десятков нанометров до единиц микрон [3].

Для генерации КЛ достаточна энергия электронов, сравнимая с шириной запрещенной зоны твердого тела (единицы электронвольт). Однако в полупроводниках и полупроводниковых структурах электронно-дырочные пары, образовавшиеся при торможении первичных электронов, могут диффундировать. Диффузия электроннодырочных пар в наногетероструктурах имеет направленный характер и определяется дизайном структур. В связи с этим область генерации КЛ равна или больше области торможения электронного пучка. Эта область может варьироваться от десятка нанометров до нескольких микрон.

\section{3. Результаты исследования и их обсуждение}

Состав верхнего слоя определялся методом РСМА при энергии электронного пучка 5 кэВ. При такой энергии глубина проникновения электронов менее толщины верхнего слоя. В табл. 1 приведены составы верхнего
Таблица 1.

\begin{tabular}{c|c|c|c}
\hline Образец & Состав НС & $\begin{array}{c}\text { Глубина } \\
\text { залегания } \\
\mathrm{HC}, \text { нм }\end{array}$ & $\begin{array}{c}\text { Состав } \\
\text { верхнего слоя }\end{array}$ \\
\hline$\# 267$ & $\mathrm{Zn}_{0.82} \mathrm{Cd}_{0.18} \mathrm{Se}$ & $241 \pm 15$ & $\mathrm{Zn}_{0.9} \mathrm{Mg}_{0.1} \mathrm{~S}_{0.22} \mathrm{Se}_{0.78}$ \\
$\# 268$ & $\mathrm{Zn}_{0.78} \mathrm{Cd}_{0.22} \mathrm{Se}$ & $303 \pm 20$ & $\mathrm{Zn}_{0.9} \mathrm{Mg}_{0.1} \mathrm{~S}_{0.2} \mathrm{Se}_{0.8}$
\end{tabular}

слоя ZnMgSSe для структур \#267 и \#268. Состав выполнялся при энергии электронного пучка 5 кэВ. Энергия электронного пучка выбиралась таким образом, чтобы область генерации характеристического рентгеновского излучения находилась внутри анализируемого слоя. На рис. 2 показана область генерации рентгеновского характеристического излучения для данной структуры.

Как было показано ранее [3], метод РСМА позволяет определить глубину залегания НС и его состав в том случае, когда в НС есть элемент, отсутствующий в других слоях гетероструктуры. В исследуемых образцах таким элементом является кадмий. Важно отметить, что методом РСМА определяется относительное количество атомов в НС, поэтому для определения его состава (в данном случае, молярной концентрации кадмия) необходимо знать толщину исследуемого НС. В первом приближении для расчета состава НС его толщина предполагалась равной 7 нм, соответствующей параметрам роста.

На первом этапе данной работы была экспериментально определена величина $K$ - отношение интенсивности излучения характеристической рентгеновской линии $K \alpha 1$ кадмия в исследуемых структурах к интенсивности этой линии на эталонном слое $\mathrm{CdSe}$ при трех различных значениях энергии первичного электронного пучка: 10 , 15 и 20 кэВ. На основании значения $K$, измеренного при одном значении энергии электронного пучка, невозможно определить два параметра - глубину залегания НС и его состав. Это связано с тем, что при измене-

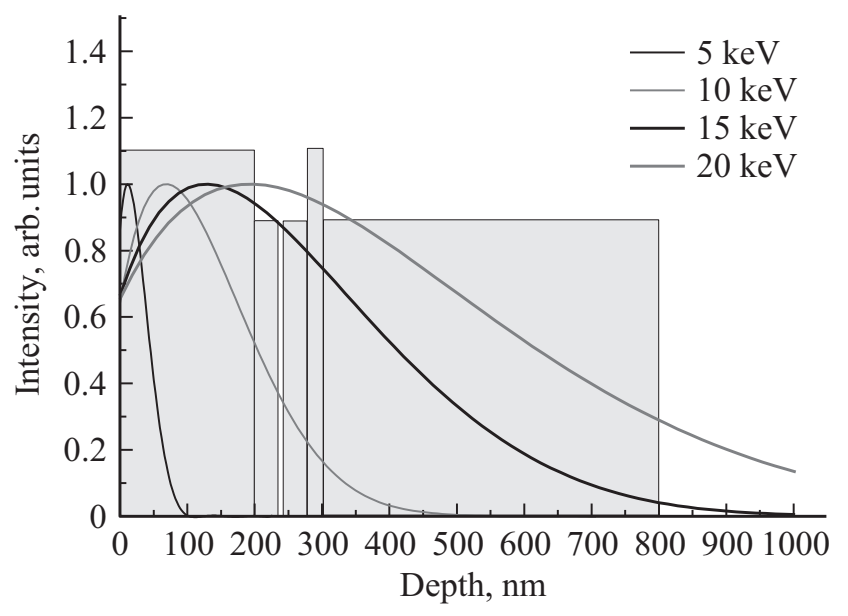

Рис. 2. Дизайн гетероструктур. Линиями указаны области генерации рентгеновского излучения. 


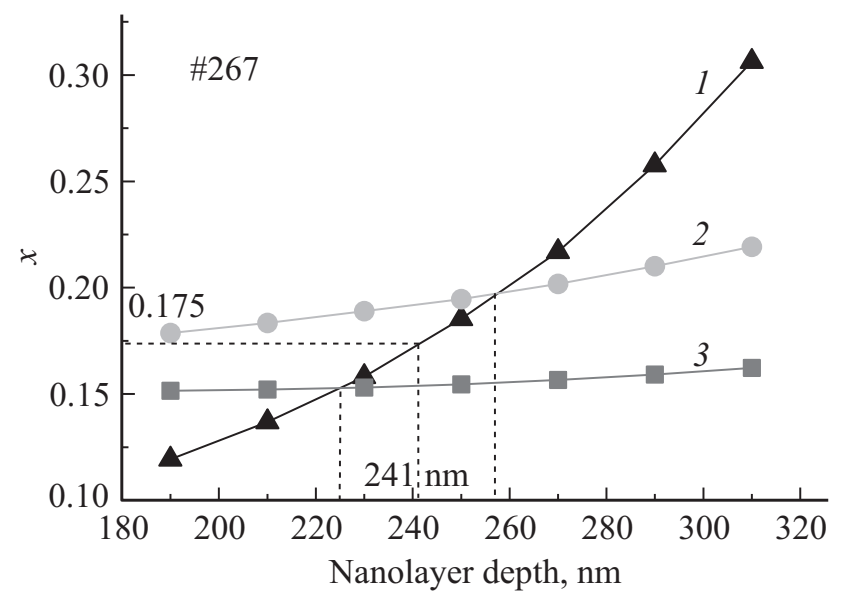

Рис. 3. Рассчитанные значения молярной концентрации $\mathrm{Cd}$ в КЯ $\mathrm{ZnCdSe}$ и глубины ее залегания при различных значениях энергии электронного пучка для гетероструктуры \#267, кэВ: $1-10,2-15,3-20$. Пунктирной линией отмечены середины допустимого диапазона значений.

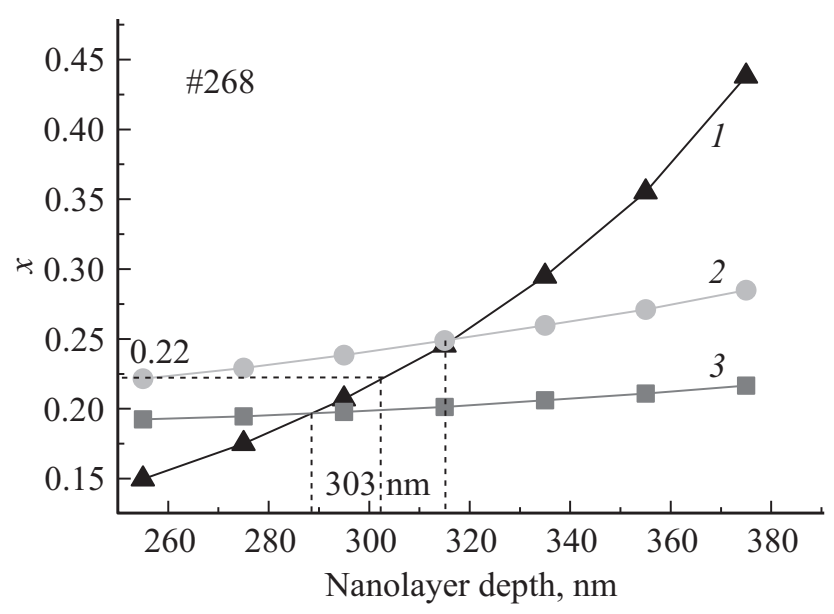

Рис. 4. Рассчитанные значения молярной концентрации $\mathrm{Cd}$ в наноразмерном слое $\mathrm{ZnCdSe}$ и глубины залегания КЯ при различных значениях энергии электронного пучка для гетероструктуры \#268, кэВ: $1-10,2-15,3-20$. Пунктирной линией отмечены середины допустимого диапазона значений.

нии глубины залегания слоя и сохранении количества кадмия величина $K$ будет различной. В связи с этим для каждого экспериментально полученного значения $K$ была рассчитана молярная концентрация кадмия в НС при различных предполагаемых глубинах его залегания. На основании рассчитанных значений была построена модельная кривая, показывающая как изменяется глубина залегания НС при изменении его состава при одном экспериментально полученном значении $K$. Для каждого образца было построено три таких модельных кривых, рассчитанных для экспериментальных значений $K$, полученных для трех различных величин энергии электронного пучка. Точка пересечения модельных кривых дает истинную глубину залегания квантовой ямы и содержание кадмия в НС (при условии, что толщина слоя равна $7 \mathrm{Hм}$ ).

На рис. 3 и 4 приведены модельные кривые, рассчитанные для трех экспериментально полученных значений $K$ для двух исследуемых структур. Как видно из рисунков, пересечение модельных кривых наблюдается не в одной точке, а в некой области, которая и определяет ошибку результатов моделирования. Величина ошибки определения глубины залегания НС определялась на основании расхождений пересечения трех модельных кривых. Эта ошибка показана на рис. 3 и 4 пунктирными линиями. Она составляет для обеих структур 10 отн\%. На рис. 3 и 4 по оси $y$ указано молярное содержание кадмия в $\mathrm{HC}$, рассчитанное на основании предположения, что его толщина равна 7 нм.

Как видно из приведенных графиков, глубина залегания НС в исследованных образцах различна. Для структуры \#268 глубина залегания НС составляет 240 нм, что хорошо согласуется с технологическими данными. Глубина залегания в образце \#268 существенно отличается от глубины, рассчитанной в соответствии с технологическими условиями роста, и составляет 300 нм. В табл. 1 приведены значения состава НС и глубины залегания слоев, полученные методом РСМА.

Определение параметров НС основывалось на спектральном положении, форме полос КЛ НС и относительном количестве атомов кадмия в слое, определенном методом РСМА.

В данной работе спектры КЛ были получены при температуре $77 \mathrm{~K}$ при энергии электронного пучка 10 кэВ. На рис. 5 приведены спектры КЛ НС для обеих исследуемых структур. Положение максимума КЛ, связанного с излучением из КЯ $\mathrm{ZnCdSe}$ (образец \#267) соответствует 2.56 эВ при ширине на полувысоте 0.034 эВ. Максимум полосы излучения НС структуры \#268 смещен в диапазон меньших энергий и наблюдается при энергии 2.24 эВ. Полуширина этой полосы больше, чем для образца \#267, и составляет 0.09 эВ. Кроме того, у этой

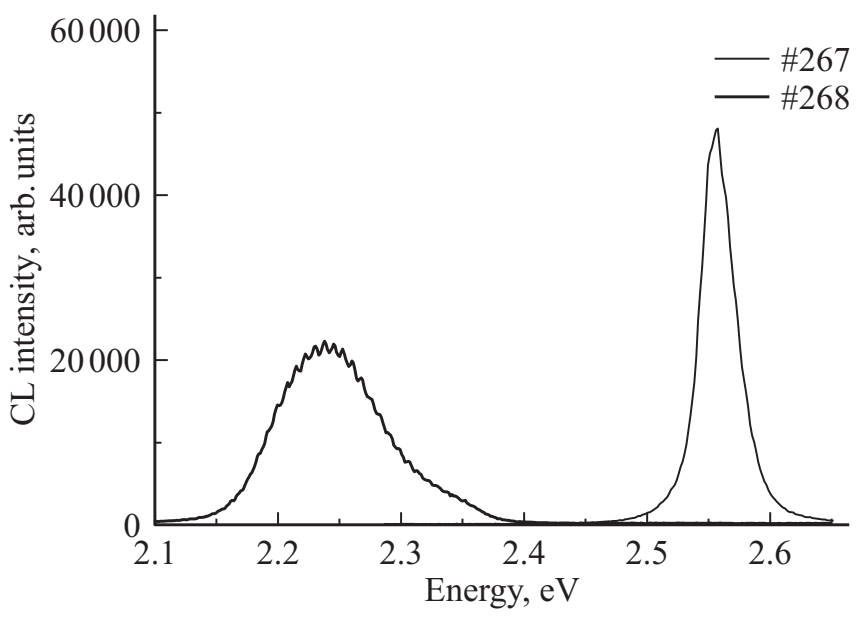

Рис. 5. Спектры КЛ наноразмерного слоя для структур \#267 и \#268. (Спектр структуры \#268 умножен на 50). 
полосы асимметричная форма, что свидетельствует либо о неоднородной толщине НС, либо о наличии локальных областей с различным содержанием кадмия.

Оценка толщины НС проводилась в предположении, что НС является квантовой ямой с составом $\mathrm{Zn}_{1-x} \mathrm{Cd}_{x} \mathrm{Se}$. Тогда положение максимума полосы КЛ может быть описано следующим выражением:

$$
E_{\mathrm{HC}}=E_{\mathrm{bulk}}^{\mathrm{ZnCdSe}}(x)+E_{e}+E_{h},
$$

где $E_{\mathrm{HC}}-$ положение максимума полосы КЛ КЯ, $E_{\text {bulk }}^{\mathrm{ZnCdSe}}(x)$ - положение полосы КЛ объемного $\mathrm{Zn}_{1-x} \mathrm{Cd}_{x} \mathrm{Se}$ того же состава, $E_{e}$ и $E_{h}-$ уровни энергии в потенциальной яме для электронов и дырок соответственно.

Как видно из выражения (1), спектральное положение максимума излучения полосы люминесценции НС зависит от положения максимума люминесценции объемного слоя того же состава и энергий уровней электронов и дырок, зависящих от толщины НС. Основной сложностью в этом случае является то, что определение состава слоя проводилось методом РСМА, при этом предполагалось, что толщина равна НС 7 нм. Изменение толщины слоя приведет к необходимости перерасчета его состава. В связи с этим оценка состава и толщины НС решалась в несколько этапов.

Первым этапом было получение численной зависимости положения полосы люминесценции объемного слоя, состав которого соответствует составу НС (первое слагаемое в выражении (1)).

Положение полосы КЛ тройного твердого раствора $\left(\mathrm{Zn}_{1-x} \mathrm{Cd}_{x}\right) \mathrm{Se}$ можно определить, основываясь на следующем выражении [4]:

$$
E_{\text {bulk }}^{\mathrm{ZnCdSe}}(x)=E_{\mathrm{bulk}}^{\mathrm{ZnSe}}+\left(E_{\mathrm{bulk}}^{\mathrm{CdSe}}-E_{\mathrm{bulk}}^{\mathrm{ZnSe}}\right) x-C_{x}(1-x),
$$

где $E_{\text {bulk }}^{\mathrm{ZnCdSe}}(x)$ - положение полосы КЛ объемного $\mathrm{Zn}_{1-x} \mathrm{Cd}_{x} \mathrm{Se}$ в зависимости от молярной концентрации $\mathrm{Cd}, E^{A B}$ - положение полосы КЛ объемного соединения $A B$ (где $A-\mathrm{Zn}$ или $\mathrm{Cd}, B-\mathrm{Se}$ ), $C-$ параметр прогиба (нелинейности зависимости спектрального положения полосы люминесценции от состава тройного раствора).

Для практического использования выражения (2) было необходимо определить коэффициент $C$ - параметр прогиба (нелинейности) для твердого раствора $(\mathrm{ZnCd}) \mathrm{Se}$. Значение параметра $C$ для данной системы в разных работах существенно отличается: 0.35 [5], 0.45 [6], 0.68 [4]. Вследствие этого, параметр $C$ был оценен в ходе выполнения данной работы. Для этого было определено спектральное положение максимумов полос КЛ $E_{\mathrm{CL}}$ для трех различных объемных слоев: $\mathrm{ZnSe}$, $\mathrm{Zn}_{0.72} \mathrm{Cd}_{0.28} \mathrm{Se}$ и $\mathrm{CdSe}$. Состав слоя $\mathrm{Zn}_{0.72} \mathrm{Cd}_{0.28} \mathrm{Se}$ был проверен методом РСМА. На основании построенной зависимости $E_{\mathrm{CL}}$ от молярной концентрации кадмия $x$ был определен коэффициент нелинейности $C$. На рис. 6 представлена полученная эмпирическая зависимость $E_{\text {bulk }}(x)$. Значение коэффициента $C$ оказалось равно 0.33 .

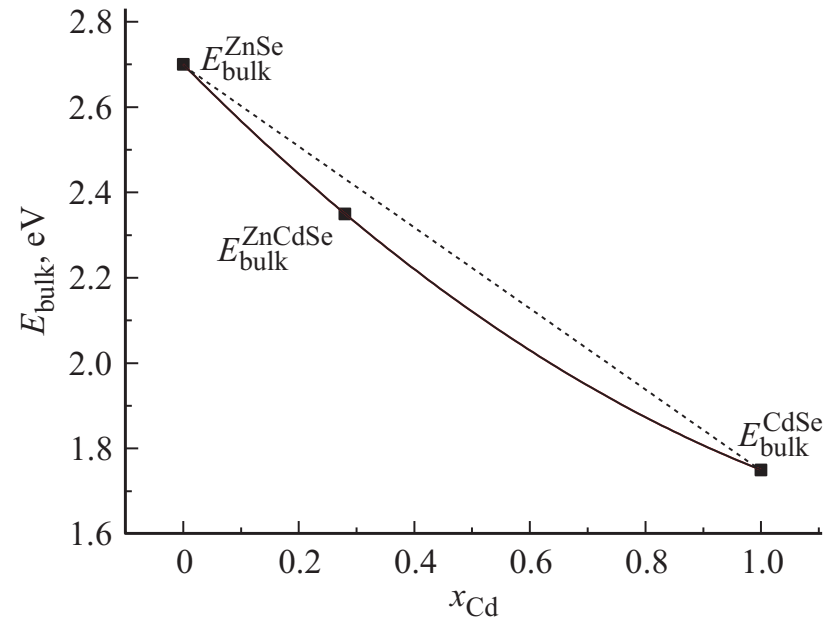

Рис. 6. Зависимость положения полосы КЛ от молярной доли кадмия в объемном слое $\mathrm{Zn}_{1-x} \mathrm{Cd}_{x} \mathrm{Se}$.

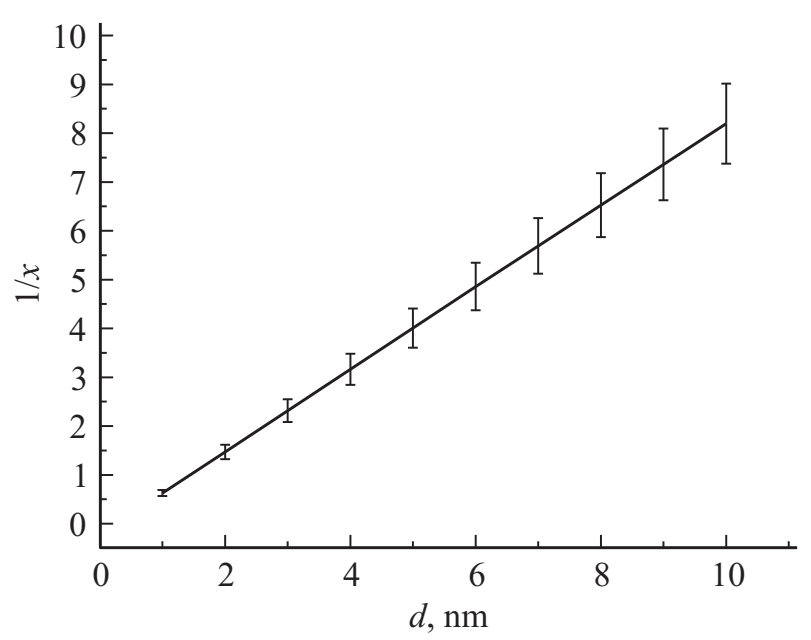

Рис. 7. Зависимость молярной доли кадмия в НС от толщины $\mathrm{HC} x(d)$.

Следующим этапом являлось построение зависимости предполагаемой молярной доли кадмия $(x)$ в НС от толщины НC $(d)$ при экспериментально определенном суммарном содержании атомов кадмия. При увеличении толщины НC молярная концентрация кадмия будет уменьшаться, потому что общее число атомов кадмия в НС постоянно. Исходя из этого, очевидно, что величина $x$ должна быть обратно пропорциональна толщине слоя $d$. В связи с этим для аппроксимации зависимости $x(d)$ использовалась функция $x^{-1}=A+B \cdot d$.

Для получения зависимости варьировалась толщина $\mathrm{HC} d$ от 3 до $10 \mathrm{Hм}$, и рассчитывалась молярная концентрация кадмия $x$. Была проведена линейная аппроксимация набора точек $1 / x_{n}, d_{n}$. Зависимость $x(d)$ отличается для слоев, находящихся на различной глубине. В связи с этим она рассчитывалась для ранее определенной методом РСМА глубины залегания слоя для обеих структур. Коэффициенты $A$ и $B$ были определены в 


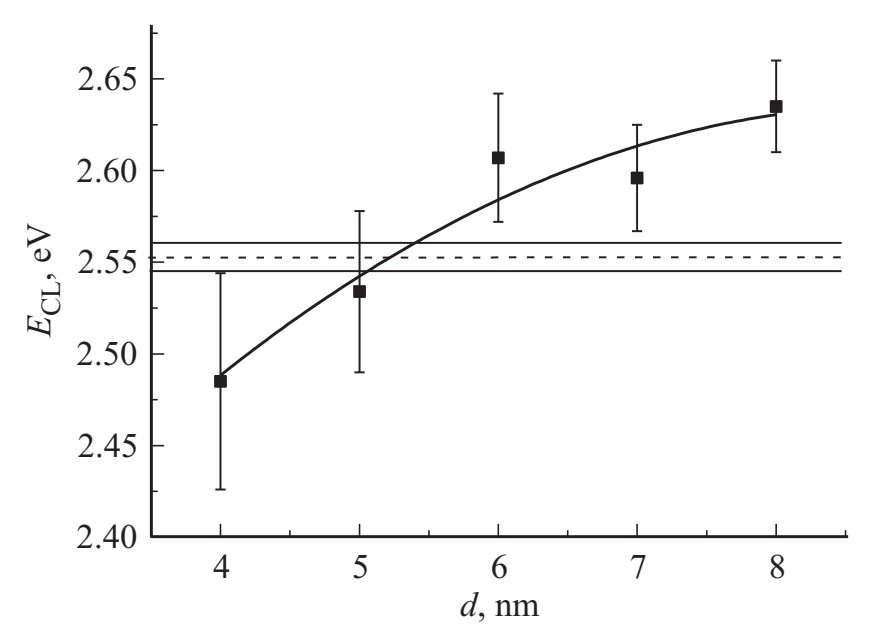

Рис. 8. График зависимости энергии полосы КЛ от толщины наноразмерного слоя для образца \#267.

ходе аппроксимации для нескольких экспериментов (при трех ускоряющих напряжениях зонда и для разных кристаллов-анализаторов). Разброс в значениях составляет не более 10 отн\%. На этом этапе были определены параметры $A$ и $B$, связывающие состав НC и его толщину. Зависимость $x(d)$ для образца \#267 приведена на рис. 7.

Далее проводилась замена $x$ в формуле (2) на полученную ранее зависимость $x(d)$.

В результате было получено следующее выражение:

$$
\begin{aligned}
E_{\mathrm{bulk}}^{\mathrm{ZnCdSe}}(d) & =E_{\mathrm{bulk}}^{\mathrm{ZnSe}}+\left(E_{\mathrm{bulk}}^{\mathrm{CdSe}}-E_{\mathrm{bulk}}^{\mathrm{ZnSe}}\right) \frac{1}{A+B \cdot d} \\
& -C^{\mathrm{ZnCdSe}} \frac{1}{A+B \cdot d}\left(1-\frac{1}{A+B \cdot d}\right) .
\end{aligned}
$$

Уровни энергии $E$ в потенциальных ямах определяются из следующего трансцендентного уравнения:

$$
\operatorname{tg}\left(\sqrt{2 m_{w} E} \frac{d}{n}\right)=\frac{2}{\sqrt{\frac{E}{U-E}}-\sqrt{\frac{U-E}{E}}},
$$

где $m_{w}$ - эффективная масса частиц в КЯ (в данной работе использовались значения для электронов $m_{e l} / m_{0}=0.16$ [7-9], для тяжелых дырок $m_{h h} / m_{0}=0.6[7,9]$, для легких дырок $\left.m_{l h} / m_{0}=0.15[8]\right)$, $E$ - уровень энергии в потенциальной яме, $d-$ толщина КЯ, $U$ - высота потенциального барьера. Высота потенциального барьера и напряжения структуры учитывались по методу, описанному в статье [6].

На рис. 8 представлены рассчитанные значения $E_{\mathrm{CL}}$ для НС разной толщины образца \#267. Пунктирная линия указывает измеренное значение пика полосы КЛ, линии обозначают полуширину полосы КЛ. Также на рис. 8 показан диапазон ошибок для значений толщины. Значение толщины ямы равно 5-6нм, в этом случае состав $\mathrm{HC}$ равен $\mathrm{Zn}_{0.75} \mathrm{Cd}_{0.25} \mathrm{Se}$.
На рис. 9 представлены графики зависимости $E_{\mathrm{CL}}(d)$ для образца \#268. Наноразмерный слой рассматривался как квантовая яма с составом $\mathrm{Zn}_{1-x} \mathrm{Cd}_{x} \mathrm{Se}$. Как видно на рис. 9, квантовая яма с измеренным составом не может давать люминесценцию на данной длине волны, поэтому было сделано предположение, что в данном образце наноразмерный слой представляет собой квантовые точки.

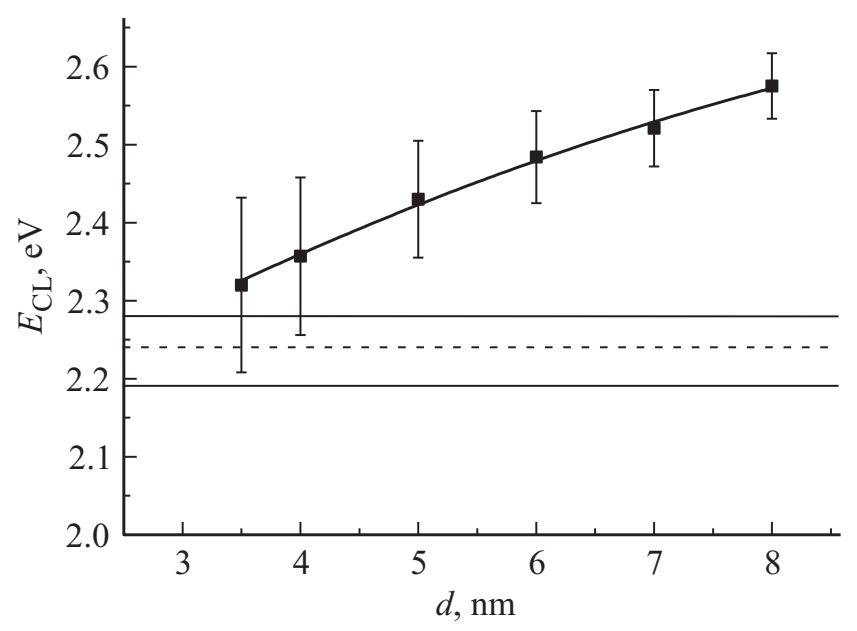

Рис. 9. График зависимости энергии полосы КЛ от толщины наноразмерного слоя для образца \#268.

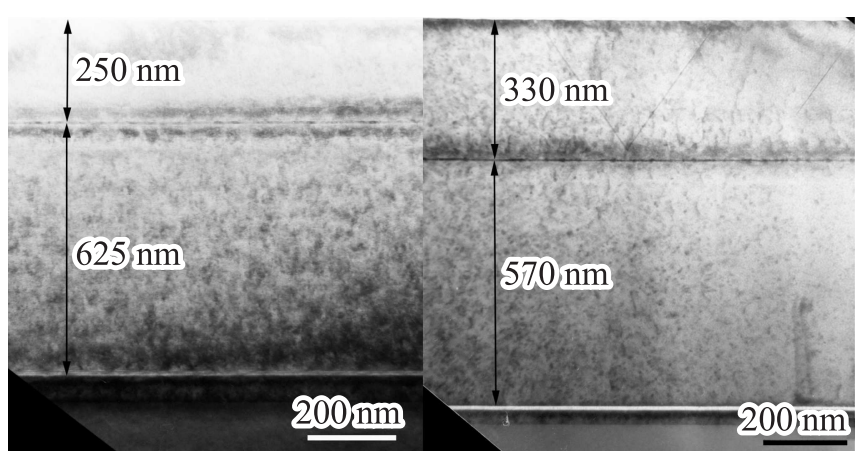

Рис. 10. Изображения поперечного сечения образца \#267(слева) и \#268(справа), полученные методом ПЭМ.
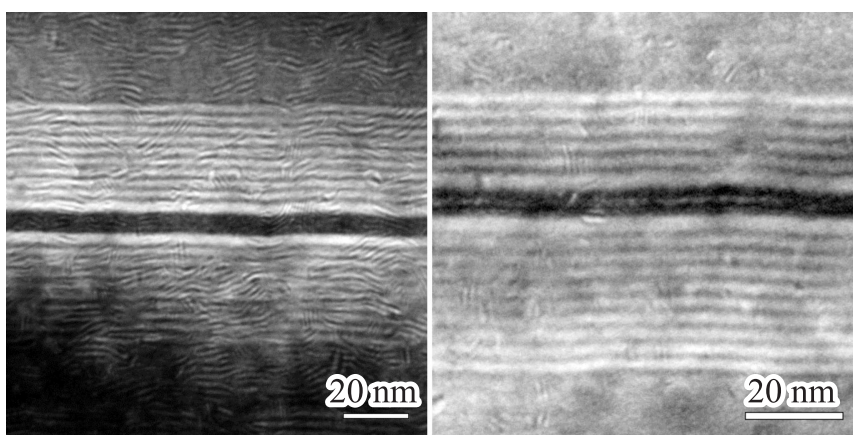

Рис. 11. Изображения НС образца \#268(справа), полученные методом ПЭМ. 
Таблица 2.

\begin{tabular}{|c|c|c|c|c|}
\hline \multirow[t]{2}{*}{ Образец } & \multirow[t]{2}{*}{ Название слоя } & \multicolumn{2}{|c|}{$\begin{array}{c}\text { Экспериментально } \\
\text { определенная толщина, нм }\end{array}$} & \multirow{2}{*}{$\begin{array}{c}\text { Технологически } \\
\text { заявленная } \\
\text { толщина, нм }\end{array}$} \\
\hline & & ПЭМ & РСМА и КЛ & \\
\hline \multirow{6}{*}{ \#267 } & Верхний слой ZnMgSSe & $206 \pm 15$ & \multirow{2}{*}{240} & 220 \\
\hline & $\mathrm{CP} \mathrm{ZnSe} / \mathrm{ZnSSe}$ & 35 & & 31.2 \\
\hline & $\mathrm{HC} \mathrm{ZnCdSe}$ & 6 & $5-6$ & 7 \\
\hline & Cp ZnSe/ZnSSe & 35 & & 31.2 \\
\hline & Барьерный слой ZnMgSSe & 25 & & 20 \\
\hline & CP ZnSe/ZnSSe & 560 & & 500 \\
\hline \multirow{6}{*}{$\# 268$} & Верхний слой ZnMgSSe & $268 \pm 20$ & \multirow{2}{*}{300} & 220 \\
\hline & CP ZnSe/ZnSSe & 20 & & 31.2 \\
\hline & $\mathrm{HC} \mathrm{ZnCdSe}$ & $3-3.5$ & $2-4$ & 7 \\
\hline & CP ZnZe/ZnSSe & 30 & & 31.2 \\
\hline & Барьерный слой ZnMgSSe & 25 & & 20 \\
\hline & $\mathrm{CP} \mathrm{ZnSe} / \mathrm{ZnSSe}$ & 520 & & 500 \\
\hline
\end{tabular}

Так как КЛ полоса НС имеет большую полуширину, можно предположить, что квантовые точки варьируются по составу и(или) по размеру.

Таким образом, такой метод расчета позволяет оценить толщину НС по спектральному положению максимума излучения данного слоя и данным РСМА. Такой метод расчета может применяться только в случае, если НС является квантовой ямой.

Для верификации результатов, полученных методами КЛ и РСМА, структуры были исследованы методом просвечивающей электронной микроскопии (ПЭМ).

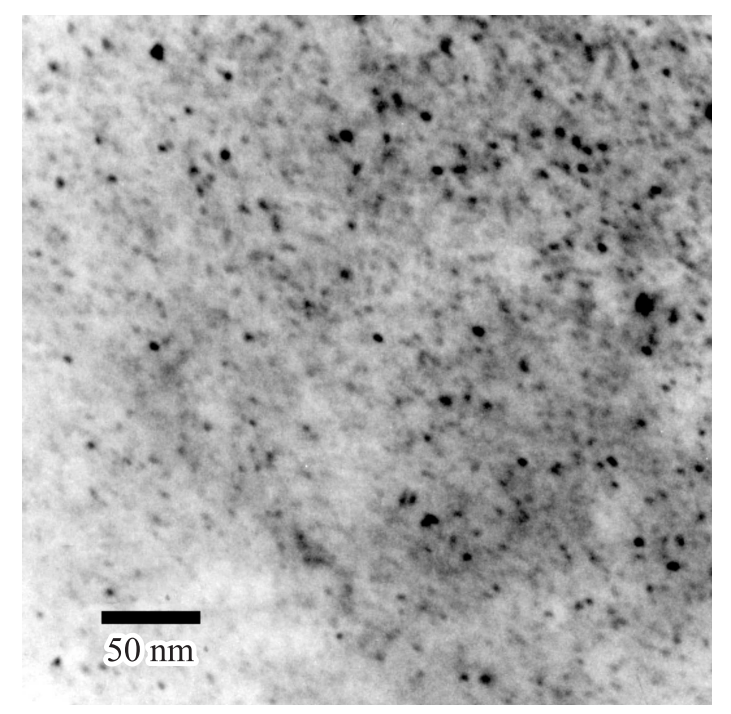

Рис. 12. Планарные изображения образца \#268, полученные методом ПЭМ.

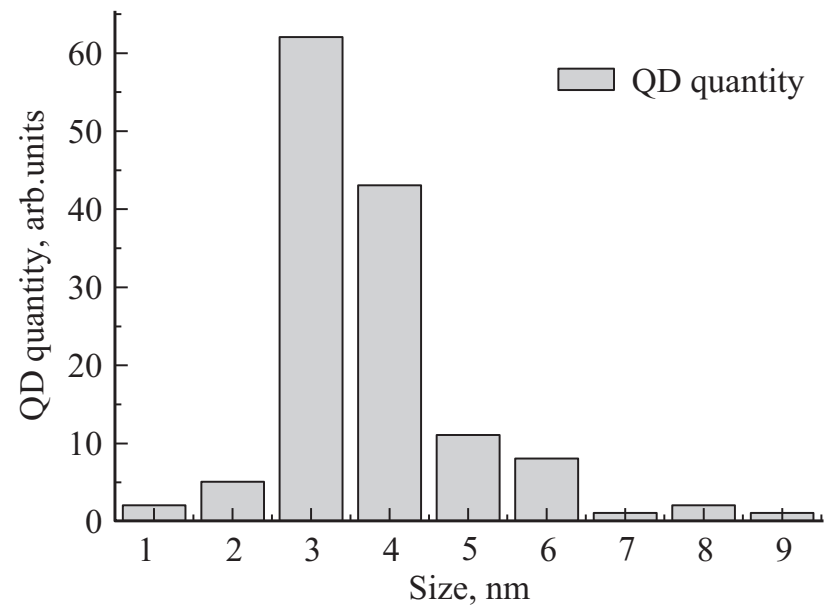

Рис. 13. Гистограмма размеров КТ для образца \#268, полученные методом ПЭМ.

На рис. 10. представлены светлопольные изображения структур \#267 и \#268, полученные в геометрии поперечного сечения. Это означает, что контраст на изображении ПЭМ формируется за счет рассеивания и поглощения электронов образцом. Области образца с большей толщиной и бо́льшим атомным номером выглядят темнее. Следует отметить, что для обеих структур толщина верхнего слоя, полученная методом РСМА, находится в хорошем согласии с толщиной, полученной методом ПЭМ. На рис. 11 видно, что в образце \#267 НС представляет собой квантовую яму, а в образце \#268 НC имеет различную толщину в разных местах, что говорит о присутствии квантовых точек разного размера. 
В табл. 2 представлены экспериментально определенные значения толщин слоев структур, определенных методами РСМА, КЛ и ПЭМ, в сравнении с толщинами, заявленными на основании технологических условий получения структур.

Также были получены изображения планарных сечений для структуры \#268. На изображении отчетливо наблюдаются квантовые точки. Из гистограммы для данной структуры следует, что средний размер КТ находится в диапазоне от 2 до 4 нм. Изображение планарных сечений и гистограмма представлены на рис. 12 и 13 соответственно.

\section{4. Заключение}

В данной работе продемонстрированы возможности локальных методов исследования гетероструктур. Было показано, что совместное использование методов РСМА и КЛ позволяет оценить глубину залегания, состав и толщину наноразмерного слоя с точностью до 10 отн\%. Рассчитанные значения толщины и глубины залегания наноразмерного слоя были подтверждены методом просвечивающей электронной микроскопии.

Работа выполнена с использованием оборудования ЦКП „Материаловедение и диагностика в передовых технологиях“ и поддержана Министерством образования и науки России (Соглашение о предоставлении субсидии № 14.621.21.0007 id RFMEFI62114Х0007). Рост структур методом МПЭ выполнен при финансовой поддержке Российского научного фонда (проект № 14-22-00107).

\section{Список литературы}

[1] М.В. Заморянская, С.Г. Конников, А.Н. Заморянский. Приборы и техника эксперимента, 3, 1 (2004).

[2] D. Drouin, A.R. Couture, D. Joly, X. Tastet, V. Aimez, R. Gauvin. Scanning, 29, 92 (2007).

[3] Т.Б. Попова, Л.А. Бакалейников, М.В. Заморянская, Е.Ю. Флегонтова. ФТП, 42 (6), 686 (2008).

[4] H. Okuyama, Y. Kishita, A. Ishibashi. Phys. Rev. B, 57, 2257 1998.

[5] V. Pellegrini, R. Atanasov, A. Tredicucci, F. Beltram. Phys. Rev. B, 51 (8), 5171 (1995).

[6] М.В. Максимов, И.Л. Крестников, С.В. Иванов, Н.Н. Леденцов, С.В. Сорокин. ФТП, 31 (8), 939 (1997).

[7] R. Chingolani, P. Prete, D. Greco, P.V. Guigno, M. Lomascolo, R. Rimaldi, L. Calcagnile, L. Vanzetti, L. Sobra, A. Franciosi. Phys. Rev. B, 51, 5176 (1995).

[8] Y. Wu, K. Ichino, Y. Kawakami, S. Fujita. Jpn. J. Appl. Phys. 31, 1737 (1992).

[9] H.J. Lozykowski, V.K. Shastri. J. Appl. Phys., 69, 3235 (1991).

Редактор Г.А. Оганесян

\section{The investigation of nanolayer properties in $A^{\mathrm{II}} \mathrm{B}^{\mathrm{VI}}$ - based nanogeterostructures}

\author{
M.B. Karavaev, D.A. Kirilenko, E.V. Ivanova, \\ T.B. Popova, A.A. Sitnikova, I.V. Sedova, \\ M.V. Zamoryanskaya \\ loffe Institute \\ 194021 St. Petersburg, Russia
}

Abstract We report on the results of a comprehensive study of $\mathrm{ZnSe}$-based nanogeterostructures grown by molecular beam epitaxy by using electron microprobe analysis and local cathodoluminescence techniques. These non-destructive methods allow determining true depth and geometrical parameters of $\mathrm{ZnCdSe}$ nanoscale. The validity of the study results was monitored by transmission electron microscopy. The research method is based on changing the $x$-ray characteristic emission and cathodoluminescence generation areas by varying the primary energy of the electron beam. 